\title{
Competencias digitales y desempeño docente en la Universidad Nacional del Centro del Perú
}

\author{
Aníbal Huachos Pacheco \\ ahuachos@uncp.edu.pe•https://orcid.org/0000-0002-4545-3527 \\ Rocío Veli Chuquillanqui \\ rcguquillanqui@uncp.edu.pe•http://orcid.org/0000-0003-2087-0890 \\ Sandy Emma Peña Ortiz \\ sandypenaortiz@gmail.com •http://orcid.org/0000-0003-4556-5593 \\ Karla Madeleyn Huachos Borja \\ e_2016200872k@uncp.edu.pe • http://orcid.org/0000-0002-4510-4714
}

\section{Resumen}

Se ha iniciado la investigación con la interrogante ¿Cuál es el tipo de asociación que existe entre las competencias digitales y el desempeño de los docentes en la Universidad Nacional del Centro del Perú?, el propósito es determinar el tipo de relación que existe entre las competencias digitales y el desempeño de los docentes de la Universidad Nacional del Centro del Perú, como posible respuesta se planteó que existe una asociación directa y significativa entre las dos variables de estudio; la investigación es de tipo básica, de nivel descriptivo, para el desarrollo de la investigación se utilizó el método científico con un diseño descriptivo correlacional, con una muestra de sujetos voluntarios conformada por 58 docentes, se recolectaron los datos con el cuestionario de competencias digitales y la encuesta de desempeño docente, al final se estableció que existe una asociación de tipo directa y alta (Tau-b=0,7101) el cual se confirma con la prueba de significancia donde el p valor $(0,0312)$ es menor que el nivel de significancia $(0,05)$, en conclusión se ha podido establecer que existe una asociación de tipo directa y significativa entre las competencias digitales y el desempeño de los docentes de la Universidad Nacional del Centro del Perú.

Palabras clave: Competencia digital, desempeño docente, universidad.

\begin{abstract}
The research has begun with the question What is the type of association that exists between digital skills and the performance of teachers at the National University of Central Peru in the semester 2020-II?, as a possible answer it was raised that there is a direct and significant association between the two study variables, the purpose of this research was to establish the type of association that exists between digital skills and the performance of teachers at the National University of Central Peru in the semester 2020 - II; for the development of the research, the scientific method was used with a descriptive correlational design, with a sample of voluntary subjects made up of 58 teachers, the data were collected with the questionnaire of digital competences and the survey of teaching performance, in the end it was established that there is an association of direct and high type (Tau-b $=0.7101)$ which is confirmed with the significance test where the $p$ value $(0.0312)$ is less than the significance level (0.05).
\end{abstract}

Keywords: Digital competence, teaching performance, university. 


\section{Introducción}

Actualmente la educación superior universitaria ha sufrido cambios significativos a partir de la vigencia de la nueva Ley Universitaria Nro. 30220; donde la exigencia es la formación de profesionales competentes que solucionen los problemas de la realidad utilizando sus capacidades, conocimientos y actitudes; los centros de formación universitaria asumen la responsabilidad de asegurar un buen aprendizaje de los estudiantes; además de su desarrollo integral.

De acuerdo con (Gonzáles, 2017) en su tesis de maestría, desarrollo un estudio que implicaba el reconocimiento, averiguación y profundización de la competencia digital que adquiere el docente dentro de su formación profesional, y de cómo ello optimiza el proceso de enseñanza, por medio de una adecuada integración de las Tecnologías de Información y Comunicación (Tics), indicando que no conocen este último término, generando problemas en función al desarrollo de las competencias digitales, de forma instrumental y metodológica, lo cual permitirá que, enfrenten los retos educativos actuales.

La problemática se forja porque existe una carencia de formación de profesionales en la docencia, que han tenido un desarrollo inadecuado de la competencia digital, sin darle importancia a una herramienta crucial para estos tiempos de desarrollo de la ciencia y la tecnología.

En otra vertiente, la sociedad de la información y del conocimiento en la cual se encuentran sumergidos los docentes universitarios, se caracteriza porque se encuentra en permanente cambio, lo cual implica desarrollar nuevas competencias, en este caso las digitales, que se basan en el uso de las Tics; lo que conlleva necesariamente que un docente universitario debe de contar fundamentalmente con una computadora personal y tener acceso a internet; lo cual favorece la motivación, interés, que se refleja en el rendimiento de sus estudiantes; además brinda un aprendizaje de calidad y un desarrollo de la autonomía, creando ambientes de aprendizaje actuales.

Por la anterior mencionado nos hemos planteado como problema general:

¿Cuál es el tipo de relación que existe entre las competencias digitales y el desempeño docente en la Universidad Nacional del Centro del Perú?

Los problemas específicos son los siguientes:

¿Cuál es el tipo de relación que existe entre las competencias digitales y la planificación del trabajo docente en la Universidad Nacional del Centro del Perú?

¿Cuál es el tipo de relación que existe entre las competencias digitales y el empleo de recursos educativos virtuales de los docentes de la Universidad Nacional del Centro del Perú?

¿Cuál es el tipo de relación que existe entre las competencias digitales y la organización del tiempo de los docentes de la Universidad Nacional del Centro del Perú?

En la actualidad, el desempeño de los docentes en las universidades, nos indica que la dimensión pedagógica constituye lo primordial del profesionalismo, ya que exige la capacidad de promover la disposición, y el compromiso con el aprendizaje de los estudiantes y su formación profesional, teniendo en cuenta en este proceso la ética, vinculando a la educación y en el crecimiento y libertad del estudiante como sujeto de la educación.

El objetivo general que ha guiado nuestra investigación es:

Determinar el tipo de relación que existe entre las competencias digitales y el desempeño docente en la Universidad Nacional del Centro del Perú.

Los objetivos específicos propuestos son los siguientes: 
Determinar el tipo de relación que existe entre las competencias digitales y la planificación del trabajo docente en la Universidad Nacional del Centro del Perú.

Determinar el tipo de relación que existe entre las competencias digitales y el empleo de recursos educativos virtuales de los docentes de la Universidad Nacional del Centro del Perú.

Determinar el tipo de relación que existe entre las competencias digitales y la organización del tiempo de los docentes de la Universidad Nacional del Centro del Perú.

Los trabajos que nos han servido de antecedentes son los que detallamos a continuación:

Esteve, 2016 en su tesis doctoral de la Universidad Rovira i Virgili, Tarragona, España, Análisis de la autopercepción y evaluación del desempeño de los estudiantes universitarios de educación por medio de un entorno $3 D$, el estudio considero comprobar la autopercepción del docente por los estudiantes en base a un entorno 3D, investigación básica, descriptiva explicativa con un diseño descriptivo simple, a una muestra probabilística de 450 alumnos se les aplico un cuestionario digital de percepción y evaluación, el estudio concluyo que los alumnos tienen una adecuada autopercepción y moderada evaluación del desempeño de los docentes universitarios.

El trabajo apoyará a la investigación porque a través de la variable de estudio desempeño docente en un entorno virtual $3 \mathrm{D}$, guiará la investigación para poder entender el desempeño como una competencia digital que tienen los docentes universitarios.

Bustos, 2017 en su trabajo de grado para maestría en el Instituto de Monterrey, Naucalpan, México, Las competencias digitales en los docentes de educación media superior, su finalidad fue determinar las competencias que tiene el docente al momento de publicar y compartir sus materiales didácticos en espacios virtuales de aprendizaje; trabajo descriptivo con diseño descriptivo simple, aplico el cuestionario de competencias digitales a una muestra estratificada de 360 docentes; arriba a concluir que los docentes presentan deficiencias en las capacidades de formación en Tics, muestran buenas habilidades al momento de manejar las herramientas tecnológicas y una destreza para operar sus equipos celulares, laptops y las pizarras digitales.

Investigación muy importante, porque muestra la base teoría de nuestra primera variable y nos ayudará para fundamentar nuestro marco teórico metodológico.

Molina, 2015 realizo su tesis de maestría de la Universidad Rafael Landívar en Quetzaltenango, Utilización de herramientas informáticas básicas para mejorar el desempeño docente, el objeto del estudio del estudio radico en establecer el nivel de conocimiento y manejo de herramientas informáticas que logren mejorar su desempeño docente, investigación aplicada, cuasi experimental, de diseño preexperimental; planteó un programa de uso de las herramientas tecnológicas a una muestra de 150 docentes, evalúo sus resultados con un cuestionario de competencias digitales, concluyendo determinar que un uso frecuente de las equipos digitales contribuye para que el docente logre que el estudiante mejore su aprendizaje.

Dicho estudio es fundamental, porque nos proporciona buena información, la cual nos guiará en la redacción de nuestro marco teórico de la variable desempeño docente.

Cueva, 2016 en su tesis de maestría de la Universidad de Guayaquil, Ecuador, El Tics y el desempeño docente en el Colegio Fiscal María Eugenia de Ruperti, del Cantón Paján, Provincia de Manabí, año 2012. Diseño de un sistema informático de capacitación para docentes, con esta investigación se encontró que tanto el estudiante como el docente sea consiente en que utilizar una serie de herramientas que involucren a las Tics durante el proceso de enseñanza resulta ser fundamental, a partir de ello resulta necesario la implementación de 
una propuesta que logre diseñar y desarrollar un sistema de capacitación que contribuya en la instrucción del docente respecto a las Tics.

San Nicolás, K. , Fariña, D. y Moreura, P., 2014 en su tesis de maestría de la Universidad de Laguna en Tenerife, España, Competencias digitales del profesorado y alumnado en el desarrollo de la docencia virtual, con esta investigación se encontró que el docente participante en la presente investigación expreso contar con una serie de competencias tanto básicas como generales sobre en qué consiste las TICs y como se pueden usar. Se trata de una competencia instrumental que contribuya a la utilización de otras herramientas que involucren las Tics que sean empleados por el docente; ejemplo de ello es la implementación de un aula virtual.

Perlaza, 2019 para su tesis de maestría en la Universidad Cesar Vallejo, Influencia de las competencias digitales para el desempeño docente en una unidad educativa Cumandá, Chimborazo, Ecuador - 2018; tuvo como objetivo determinar con la finalidad de determinar la influencia entre las competencias digitales y el desempeño docente de los docentes de la "Unidad Educativa Sultana de los Andes" del cantón Cumandá Provincia de Chimborazo, Ecuador 2018; de tipo básica, descriptiva, con diseño correlacional, para una muestra probabilística de 15 docentes de forma intencional con el cuestionario de escala ordinal para las competencias digitales y el desempeño docente, culmino con una correlación alta de 0,806 , asumiendo que existe una buena relación directa entre sus variables de estudio.

La tesis nos apoyara en la discusión de los resultados, ya que tiene el mismo tipo de instrumentos que vamos a utilizar para la toma de datos en nuestra muestra y los resultados de tipo ordinal coadyubaran en el apoyo de las conclusiones y recomendaciones.

Torres, 2019 tesis de maestría Universidad Cesar Vallejo, Las competencias y el desempeño docente en la institución educativa "Felipe Santiago Estenos", UGEL 06, 2018.el trabajo tuvo como objetivo determinar la relación que existe entre las competencias digitales y el desempeño docente. obedece a un enfoque cuantitativo, de diseño no experimental, correlacional y de corte transversal; con una muestra que comprende 96 docentes, a quienes se les aplicó cuestionarios, y se utilizó el coeficiente de correlación de Spearman para la prueba de hipótesis. Se determinó que existe una relación significativa y moderada entre las competencias digitales y el desempeño docente en la institución educativa "Felipe Santiago Estenos", Ugel 06, 2018; habiéndose obtenido una rho de Spearman $=0,491$ y un p-valor $=0,000$.

Escobedo, R. y Solorzano, J., 2018 con la tesis de maestría Universidad Cesar Vallejo, "Competencias Digitales Y La Práctica Docente En La Universidad Nacional Diego Quispe Tito De Cusco", cuyo objetivo fue el de comparar y evaluar el grado de conexión que existe entre las dos variables. Con una población de 35 docentes de la Universidad Nacional Diego Quispe Tito, aplicándose los instrumentos a una muestra distintiva de 22 docentes, obtenido de un muestreo no probabilístico intencionado. La compilación de datos se hizo con el cuestionario de competencias digitales y el cuestionario de práctica docente y las dos variables se analizaron mediante la $\mathrm{r}$ de Spearman. Según el análisis estadístico se logró llegar a la siguiente conclusión donde el valor estadístico de Spearman es de 0,691 mientras que sus niveles de significancia resultan $0,000(\mathrm{p}<0,005)$. Esto quiere decir que hay una correlación inmediata, moderada y demostrativa entre la competencia digital y la práctica docente; entendiéndose que el valor significativo del estadístico es mínimo que 0,05, se puede afirmar que el factor de correlación es demostrativo $(\mathrm{p}<0,05)$. En tal sentido se admite la hipótesis alternativa impugnándose la hipótesis nula. 
Alva, 2018 su tesis de maestría Universidad Cesar Vallejo, "Plataforma virtual en la competencia digital docente en la Universidad de Cañete, 2018”, con su propósito evaluar las diferencias existentes al aplicar la plataforma virtual en la formación de competencia digital del docente, de enfoque cuantitativo, método deductivo, investigación aplicada, con diseño pre experimental, se administró el cuestionario de desempeño docente, para una muestra no probabilística e intencional de 20 docentes, finalmente desembocó en la existencia de una diferencia significativa en la aplicación de la plataforma virtual Moodle para la competencia digital docente de los integrantes de la muestra para una prueba paramétrica de Wilcoxon $(0,00<0,05)$.

Trabajo que servirá de base para orientarnos en la temática de estudio, y aportara al momento de la discusión de los resultados porque concuerdan los instrumentos de recolección de datos.

Mayuri, E., Gerónimo, C. y Ramos, A., 2016 tesis de maestría Universidad Marcelino Champagnat, "Competencias digitales y desempeño docente en el aula de innovación pedagógica de las redes educativas 03, 05 y 15 - UGEL 01; su propósito consistió en relacionar las competencias digitales y el desempeño docente, para las aulas de innovación pedagógica en las redes educativas número 03, 05 y 15, las que pertenecen a la Unidad de Gestión Educativa Local Nro. 01 de Lima, estudio aplicado, descriptivo, de diseño transeccional-correlacional, se administró la escala de competencias digitales, como también la lista de cotejo de evaluación de desempeño docente en el aula de innovación docente, a una muestra no probabilística e intencional de 111 docentes; como consecuencia del estudio se estableció que existe una correlación positiva y alta $(\mathrm{r}=0831)$ para las variables en cuestión.

El estudio nos permitirá realizar una discusión adecuada, porque trabaja con las mismas variables que se está desarrollando el presente trabajo de investigación.

\section{Competencias digitales}

Una competencia digital, para la (Comisión Europea, 2005) "es la utilización que se da de forma confiada y crítica de los sistemas tecnológicos para realizar un trabajo, esparcimiento y dialogó" (pág. 140). Nos indica que, para que una persona logre el desarrollo de las competencias digitales; es necesario que debe de contar con una adecuada comprensión y entendimiento, además de un saber amplio sobre: "la naturaleza, la función y la oportunidad de la tecnología en la sociedad de la información frente a una situación cotidiana de la vida tanto personal, social y profesional" (pág. 141).

Motivo por el cual, cuando se utilizan las Tecnologías de la Información y Comunicación, existe una interacción entre los agentes educativos en tiempo real, por lo que el acceder a la información para mejorar y afianzar su aprendizaje se saberes es de forma inmediata.

En consonancia con (Chan, 2015) indica que el "tipo de competencia necesaria para actuar dentro de los contextos digitales son llamadas mediacionales, estas contribuyen al desarrollo del pensamiento crítico, la participación, la comunicación, la tolerancia y la variedad, para producir un nuevo conocimiento y la funcionalidad del aprendizaje" (pág. 81).

Por lo que, dicho procedimiento integra gran variedad de destrezas, dentro de las cuales se tiene la accesibilidad a la información, analizando, aplicando y comunicando los resultados. En el momento de existir una valoración de la competencia desde un punto de vista actitudinal, se enfatiza como componente primordial de la autonomía a la responsabilidad, la ética para el manejo y uso adecuado de la información, como también la habilidad de analizar y sintetizar. 
Con arreglo a, Imbermón citado por (Henríquez, 2012) discurre "se necesita concretar la función del docente o la competencia profesional que necesita para poder cumplir con las necesidades educativas al estudiante y de la misma comunidad".

En concordancia a tal afirmación, todo docente comprometido con la tecnología necesita de competencias adecuadas a esta era, primordialmente las digitales, lo cual ayudará a mejorar su desempeño profesional; con lo cual aportará a la sociedad con una educación de calidad para sus estudiantes.

Desempeño docente

Son variados los términos para referirse al desempeño docente, algunos prefieren desempeño del maestro o desempeño profesional pedagógico; lo que sí es cierto es que en la actualidad es de vital importancia el estudio del desempeño de los docentes toda vez que este es un productor de la calidad del servicio que se brinda al estudiante e impacta directamente en su aprendizaje; pero sin que pierda su naturaleza que es el de permitirle al docente su desarrollo integral.

En consonancia de, Montenegro (2018) afirma que es "el cumplimiento de sus funciones; éste se halla determinado por factores asociados al docente, estudiante y entorno. También, el desempeño se ejerce en diferentes campos: el contexto sociocultural, el entorno institucional, el ambiente de aula y sobre el propio docente una actividad mediante una acción reflexiva" (pág. 264).

Es importante el desempeño docente, porque permite mejorar la calidad educativa y calificar la profesión docente.

Como desempeño se entienden todas las actividades que realizan las personas, en una determinada actividad o función humana dentro de la sociedad, ya sea esta para producir un bien o brindar un servicio.

Todo docente al momento de realizar su labor educativa debe tener en cuenta la responsabilidad, el compromiso, la tolerancia y la gestión de los valores morales, cuya finalidad es de conseguir una formación académica y científica bien sólida, en sus estudiantes.

De acuerdo con (Diaz, 2019) "es el desarrollar una buena labor dentro del salón de clases contribuyendo tanto al crecimiento de la institución como del docente profesionalmente" (pág. 16).

Ciertamente, todo docente de vocación tiene que cumplir un rol protagónico importante en la formación integral del ser humano, ya sea que se desenvuelva en cualquier nivel de educación del sistema educativo peruano.

Para (Valdez, 2017) se entiende por desempeño docente cuando "es comprendida como la realización de su labor: la cual es determinada por una serie de componentes que se encuentran vinculados al docente, estudiante y a su entorno” (pág. 154).

Con arreglo a (Rizo, 2015) se considera "procesos de movilización de su capacidad profesional, su disposición como persona y su compromiso social para lograr articulación de las relaciones significativas entre los factores que impactaron en la formación del docente; también en la participación en la gestión educativa" (pág. 148).

Según (Diaz, 2019) significa lograr "un objetivo de un suceso educativo, de manera, que el compromiso del docente es incentivar su formación de manera amplia del estudiante, yendo más allá de su destreza cognitiva, presentando funciones memorísticas o de dominios de procedimiento" (pág. 89).

Se tiene que comprender que el desempeño docente, contribuye a saber su comporta- 
miento y rendimiento en un lapso determinado, cuya finalidad es incentivar para su desarrollo institucional eficaz, y eficiente, como también su desarrollo individual el cual es su derecho.

Nos propusimos demostrar la siguiente hipótesis general:

Existe un relación directa y significativa entre las competencias digitales y el desempeño de los docentes de la Universidad Nacional del Centro del Perú

Las hipótesis especificas planteadas con las siguientes:

Existe una relación directa y significativa entre las competencias digitales y la lanificación del trabajo docente en la Universidad nacional del Centro del Perú.

Existe una relación directa y significativa entre las competencias digitales y el empleo de recursos educativos virtuales de los docentes en la Universidad Nacional del Centro del Perú.

Existe una relación directa y significativa entre las competencias digitales y la organización del tiempo en los docentes de la Universidad Nacional del Centro del Perú.

\section{Metodología}

El trabajo de investigación es una investigación de tipo básica (Sánchez, H. y Reyes, C., 2015) porque busca conocer la relación que existe entre las competencias digitales y el desempeño de los docentes de la Universidad Nacional del Centro del Perú.

De manera similar tomando como referencia a (Sánchez, H. y Reyes, C., 2015) la investigación pertenece al nivel descriptivo correlacional, ya que se describen y relacionan las competencias digitales y el desempeño de los docentes.

El diseño de investigación es no experimental, es el descriptivo correlacional (Sánchez, H. y Reyes, C., 2015); cuyo esquema es el siguiente:

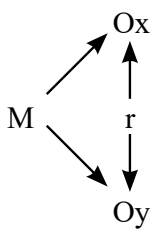

La población estuvo conformada por 689 docentes de las diferentes facultades de la Universidad Nacional del Centro del Perú.

El muestreo fue no probabilístico de tipo sujetos voluntarios, conformado por 58 docentes quienes respondieron los instrumentos de investigación.

Según Sánchez y Reyes (2015) sostienen que, "el muestreo voluntario es un tipo de muestreo no probabilístico, formado por participantes seleccionados por el investigador. Sin embargo, este método produce un sesgo de respuesta, ya que los miembros son autoseleccionados" (pág. 125).

La técnica fue la encuesta y los instrumentos fueron el cuestionario de competencias digitales y la encuesta de apreciación estudiantil.

En concordancia con Sánchez y Reyes (2015) afirman que, "la encuesta es una técnica que se lleva a cabo mediante la aplicación de un cuestionario a una muestra de personas. Las encuestas proporcionan información sobre las opiniones, actitudes y comportamientos de los ciudadanos" (pág. 168). 


\section{Resultados}

Nuestra muestra la conformaron 58 docentes, quienes contestaron el cuestionario de competencias digitales y la encuesta de evaluación docente; los resultados los explicamos y analizamos a continuación.

\section{Tabla 01}

Categorías de las competencias digitales de los docentes de la Universidad Nacional del Centro del Perú en el 2020 - II

\begin{tabular}{lccc}
\hline & Niveles & $\mathrm{Fi}$ & $\%$ \\
\hline Bajo & & 4 & $6.9 \%$ \\
Medio & & 24 & $41.4 \%$ \\
Alto & 30 & $51.7 \%$ \\
\hline & Total & 58 & $100.0 \%$ \\
\hline
\end{tabular}

Fuente: procesamiento del cuestionario con el programa R del Microsoft Excel 2019

En la tabla 01 se aprecia que, de los docentes encuestados, 4 (6,9\%) de ellos presentan un nivel bajo en sus competencias docentes, 24 (41,4\%) evidencian un nivel medio y $30(51,7 \%)$ han evidenciado un nivel alto.

\section{Tabla 02}

Categorías del desempeño de los docentes de la Universidad Nacional del Centro del Perú en el 2020 - II

\begin{tabular}{lccc}
\hline & Niveles & $\mathrm{Fi}$ & $\%$ \\
\hline Bajo & & 8 & $13.8 \%$ \\
Medio & 22 & $37.9 \%$ \\
Alto & 28 & $48.3 \%$ \\
\hline & Total & 58 & $100.0 \%$
\end{tabular}

Fuente: procesamiento del cuestionario con el programa R del Microsoft Excel 2019

En la tabla 02 , se puede evidenciar que de los docentes de la muestra que han respondido los cuestionarios, $8(13,8 \%)$ presenta un nivel de desempeño docente bajo, 22 (37,9\%) medio y $28(48,3 \%)$ mostraron un nivel alto.

\section{Tabla 03}

Correlación del nivel de competencias digitales con el nivel de desempeño docente

\begin{tabular}{|c|c|c|c|c|}
\hline & & & $\begin{array}{c}\text { Competencias } \\
\text { digitales }\end{array}$ & $\begin{array}{c}\text { Desempeño } \\
\text { docente }\end{array}$ \\
\hline \multirow{6}{*}{$\begin{array}{l}\text { Tau_b de } \\
\text { Kendall }\end{array}$} & \multirow{3}{*}{$\begin{array}{l}\text { Competencias } \\
\text { digitales }\end{array}$} & Coeficiente de correlación & 1,000 &, 8164 \\
\hline & & Sig. (bilateral) & . & ,0092 \\
\hline & & $\mathrm{N}$ & 58 & 58 \\
\hline & \multirow{3}{*}{$\begin{array}{l}\text { Desempeño } \\
\text { docente }\end{array}$} & Coeficiente de correlación & 8164 & 1,000 \\
\hline & & Sig. (bilateral) & ,0092 & \\
\hline & & $\mathrm{N}$ & 58 & 58 \\
\hline
\end{tabular}

Fuente: Procesador SPSS V25 
En la tabla 03 encontramos que se tiene un coeficiente de correlación de 0,8164 que nos indica que la asociación entre el nivel de competencias digitales y desempeño de los docentes es de tipo alta y directa, lo que se reafirma con la prueba de significancia donde el p valor $(0.0092)$ es menor que el nivel de significancia $(0,05)$.

\section{Tabla 04}

Correlación del nivel de las competencias digitales y la planificación del trabajo docente

\begin{tabular}{|c|c|c|c|c|}
\hline & & & $\begin{array}{c}\text { Competencias } \\
\text { digitales }\end{array}$ & $\begin{array}{l}\text { Planificación del } \\
\text { trabajo docente }\end{array}$ \\
\hline \multirow{6}{*}{$\begin{array}{l}\text { Tau_b de } \\
\text { Kendall }\end{array}$} & \multirow{3}{*}{$\begin{array}{l}\text { Competencias } \\
\text { digitales }\end{array}$} & Coeficiente de correlación & 1,000 & ,7101 \\
\hline & & Sig. (bilateral) & . & 0312 \\
\hline & & $\mathrm{N}$ & 58 & 58 \\
\hline & \multirow{3}{*}{$\begin{array}{l}\text { Planificación del } \\
\text { trabajo docente }\end{array}$} & Coeficiente de correlación & ,7101 & 1,000 \\
\hline & & Sig. (bilateral) &, 0312 & \\
\hline & & $\mathrm{N}$ & 58 & 58 \\
\hline
\end{tabular}

Fuente: Procesador SPSS V25

En la tabla 04 podemos comprobar que la correlación entre el nivel de competencias digitales y la planificación del trabajo docente es positiva y alta con un coeficiente de correlación de 0,7101 , lo que se confirma con el p valor $(0,0312)$ es menor que el nivel de significancia $(0,05)$.

Tabla 05

Correlación del nivel de competencias digitales y el empleo de recursos educativos virtuales

\begin{tabular}{|c|c|c|c|c|}
\hline & & & $\begin{array}{l}\text { Competencias } \\
\text { digitales }\end{array}$ & $\begin{array}{l}\text { Empleo de recursos } \\
\text { educativos virtuales }\end{array}$ \\
\hline \multirow{6}{*}{$\begin{array}{l}\text { Tau_b de } \\
\text { Kendall }\end{array}$} & \multirow{3}{*}{$\begin{array}{l}\text { Competencias } \\
\text { digitales }\end{array}$} & Coeficiente de correlación & 1,000 & ,7159 \\
\hline & & Sig. (bilateral) & . &, 0114 \\
\hline & & $\mathrm{N}$ & 58 & 58 \\
\hline & \multirow{3}{*}{$\begin{array}{l}\text { Empleo de } \\
\text { recursos } \\
\text { educativos } \\
\text { virtuales }\end{array}$} & Coeficiente de correlación &, 7159 & 1,000 \\
\hline & & Sig. (bilateral) &, 0114 & . \\
\hline & & $\mathrm{N}$ & 58 & 58 \\
\hline
\end{tabular}

Fuente: Procesador SPSS V25

En la tabla 05 que corresponde a la correlación del nivel de competencias digitales y el empleo de recursos educativos virtuales tiene un coeficiente de correlación de 0,7159 que nos indica que es directa y alta, lo que se reafirma con la prueba de significancia donde el $\mathrm{p}$ valor $(0,0114)$ es menor que el nivel de significancia $(0,05)$. 
Tabla 06

Correlación del nivel de competencias digitales con el nivel de organización del tiempo

\begin{tabular}{lllr|r}
\hline & & \multicolumn{1}{c}{$\begin{array}{c}\text { Competencias } \\
\text { digitales }\end{array}$} & $\begin{array}{c}\text { Organización } \\
\text { del tiempo }\end{array}$ \\
\hline \multirow{3}{*}{$\begin{array}{l}\text { Tau_b de } \\
\text { Kendall }\end{array}$} & $\begin{array}{l}\text { Competencias } \\
\text { digitales }\end{array}$ & Coeficiente de correlación & 1,000 &, 8121 \\
\cline { 2 - 5 } & Sig. (bilateral) & N & 58 &, 0237 \\
\cline { 2 - 5 } & $\begin{array}{l}\text { Organización del } \\
\text { tiempo }\end{array}$ & Coeficiente de correlación &, 8121 & 58 \\
\cline { 2 - 5 } & Sig. (bilateral) &, 0237 & 1,000 \\
\hline
\end{tabular}

Fuente: Procesador SPSS V25

En la tabla 06 hemos encontrado que el coeficiente de correlación para el nivel de las competencias digitales y la organización del tiempo es 0,8121 que nos indica una relación directa y alta, lo que se confirma con la prueba de significancia donde el p valor $(0,0237)$ es menor que el nivel de significancia $(0,05)$.

\section{Discusión}

Los resultados de la investigación demuestran que se tiene un nivel alto de competencias digitales 51,7\% para los docentes de la Universidad Nacional del Centro del Perú, lo que se confirma con el trabajo de (Bustos, 2017) quien ha obtenido en su estudio un nivel elevado para el $48,7 \%$ de los integrantes de su estudio.

Los resultados del desempeño docente en nuestra muestra han evidenciado que existe un $48,3 \%$ en el nivel alto lo que se reafirma con el estudio de (Esteve, 2016) quien obtuvo en sus resultados que un $54,78 \%$ de los integrantes de su muestra obtuvo el nivel elevado.

Los resultados nos indican que la asociación entre las competencias digitales y el desempeño docente en la Universidad Nacional del Centro del Perú tienen una asociación directa y significativa (Tau- $b=0,8164$ ), lo que es confirmado por (Molina, 2015) quien muestra una correlación positiva y moderada $(\mathrm{r}=07485)$.

Los resultados obtenidos entre las competencias digitales y la planificación del trabajo docente muestran un coeficiente de correlación (Tau- $b=0,7101)$ que nos indica que es positiva y moderada, lo que se confirma con el estudio de (Perlaza, 2019) que ha obtenido una correlación (rho=08641) que es positiva y alta.

Los resultados de la correlación del nivel de competencias digitales y el empleo de recursos educativo-virtuales tienen un coeficiente (Tau- $b=0,7159)$ que nos indica que es directa y alta, lo que es confirmado en el estudio de (Torres, 2019) que ha obtenido un coeficiente de correlación alto y directo $(\mathrm{r}=0,8267)$.

Los resultados que se han encontrado al correlacionar los niveles de competencias digitales y la organización del tiempo en los docentes de la muestra nos indican que tienen un coeficiente de correlación (Tau-b $=0,8121$ ) que sostiene que la relación es directa y alta; el cual es confirmado en la investigación de (Escobedo, R. y Solorzano, J., 2018) quienes han obtenido una correlación positiva y moderado con un coeficiente de $(\mathrm{Rho}=0,6845)$. 


\section{Conclusiones}

Se ha establecido que existe una asociación de tipo directa y significativa entre las competencias digitales y el desempeño de los docentes de la Universidad Nacional del Centro del Perú.

Se pudo establecer que la asociación que existe entre las competencias digitales y la planificación del trabajo docente es directa y alta, para los docentes de la muestra.

Se estableció que el tipo de asociación que existe entre las competencias digitales y el empleo de recursos educativos virtuales es directo y significativo alto en los docentes encuestados.

Se ha podido establecer que entre las competencias digitales y la organización del tiempo en los docentes de la muestra tienen una asociación de tipo positiva y alta.

\section{Referencias}

Alva, C. (2018). Plataforma virtual en la competencia digital docente en la Universidad de Cañete, 2018. Tesis de maestría, Universidad Cesár Vallejo, Cañete.

Bustos, M. (2017). Las competencias digitales en los docentes de educación media superior. Tesis de maestría, Instituto Tecnológico de Monterrey, Monterrey.

Chan, M. (2015). Competencias mediacionales para la educación en línea. Madrid: Navara.

Comisión Europea. (25 de Abril de 2005). Competencias medianacionales para la educación en línea. Revista Electrónica de Investigación Educativa, XII(6), 139-142. Recuperado el 2 de Marzo de 2020, de http://www.educastur.princast.es/info/calidad/indicadores/doc/comision_europea.pdf

Cueva, M. (2016). El TICs y el desempeño docente en el Colegio Fiscal María Eugenia de Ruperti, del Cantón Paján, Provincia de Manabí, año 2012. Tesis de maestría, Universidad de Guayaquil, Guayaquil.

Diaz, T. (2019). Evaluación de los docentes de formación magisterial. Ciudad de México: Mc GrwHill.

Escobedo, R. y Solorzano, J. (2018). Competencias Digitales Y La Práctica Docente En La Universidad Nacional Diego Quispe Tito De Cusco. Tesis de maestría, Universidad César Vallejo, Lima.

Esteve, K. (2016). Análisis de la autopercepción y evaluación del desempeño de los estudiantes universitarios de educación por medio de un entorno $3 D$. Tesis doctoral, Universidad Rovira $\mathrm{i}$ Virgili, Tarragona.

Henríquez, M. (2012). Formación del profesorado en tecnologías de la Información y Comunicación (Sexta ed.). Tarragona, España: Rovira i Virgili.

Mayuri, E., Gerónimo, C. y Ramos, A. (2016). Competencias digitales y desempeño docente en el aula de innovación pedagógica de las redes educativas 03, 05 y 15 - Ugel 01. Tesis de maestría, Universidad Marcelino Champagnat, Lima.

Molina, R. (2015). Utilización de herramientas informáticas básicas para mejorar el desempeño docente. Tesis de maestría, Universidad Rafael Landivar de México, Quetzaltenango.

Montenegro, P. (2018). Evaluación del desempeño docente. Bogotá: Harla.

Perlaza, C. (2019). Influencia de las competencias digitales en el desempeño docente de una unidad educativa Cumandá-Chimborazo-Ecuador 2018. Tesis de maestría, Universidad César Vallejo, Lima.

Rizo, G. (2015). Evaluando a los formadores de Educación Básica. Madrid: Harla.

San Nicolás, K. , Fariña, D. y Moreura, P. (2014). Competencias digitales del profesorado y alumnado en el desarrollo de la docencia virtual. Tesis de maestría, Universidad de Laguna España, Tenerife.

Sánchez, H. y Reyes, C. (2015). Metodología y diseños en la investigación científica. Lima: Universidad Ricardo Palma.

Torres, L. (2019). Competencias digitales y desempeño docente en la institución educativa "Felipe Santiago Estenós”, Ugel 06, 2018. Tesis de maestría, UNiversidad César Vallejo, Lima.

Valdez, F. (2017). Evaluando a los docentes de bachillerato. Barcelona: Narcea. 